CONFORMAL GEOMETRY AND DYNAMICS

An Electronic Journal of the American Mathematical Society

Volume 10, Pages 1-20 (January 10, 2006)

S $1088-4173(06) 00120-2$

\title{
GEOMETRY OF INFINITELY GENERATED VEECH GROUPS
}

\author{
PASCAL HUBERT AND THOMAS A. SCHMIDT
}

\begin{abstract}
Veech groups uniformize Teichmüller geodesics in Riemann moduli space. We gave examples of infinitely generated Veech groups; see Duke Math. J. 123 (2004), 49-69. Here we show that the associated Teichmüller geodesics can even have both infinitely many cusps and infinitely many infinite ends.
\end{abstract}

\section{INTRODUCTION}

The study of billiards on Euclidean polygonal tables has benefitted greatly from the use of the theory of Riemann surfaces. A classical unfolding process associates to each rational-angled polygonal table a Riemann surface with holomorphic 1form; see [KZ]. Using this, Kerckhoff-Masur-Smillie [KMS] showed that for almost every direction on a polygonal table, the billiard flow is ergodic. Soon thereafter, Veech Vch2 identified a setting where the only non-ergodic directions are those of saddle connections; these examples are identified by an associated group, the Veech group, being a lattice Fuchsian group. In Vch3, Veech asked if in general these groups can ever fail to be finitely generated. Only recently have examples of infinitely generated Veech groups been given [HS3, Mc2. Here we study the geometry associated to the groups of [HS3].

The Riemann moduli space $\mathcal{M}_{g}$ is the space of smooth compact Riemann surfaces of genus $g$, up to biholomorphic equivalence. The holomorphic 1-forms on a Riemann surface $X$ of genus $g$ form a $g$-dimensional complex vector space, $\Omega(X)$. Pairs $(X, \omega)$ with $\omega \in \Omega(X)$ form a bundle $\Omega \mathcal{M}_{g} \rightarrow \mathcal{M}_{g}$ (of course, $\mathcal{M}_{g}$ is not a manifold, but rather an orbifold, a fiber is actually $\Omega(X) / \operatorname{Aut}(X))$. Each pair $(X, \omega)$ (with $\omega$ non-null) identifies a translation structure on the real surface $X$ : local coordinates are defined by integration of $\omega$. Invoking the real structure of $\mathbb{C}$, there is an $\mathrm{SL}(2, \mathbb{R})$-action on these translation surfaces: post-composition with the local coordinate functions.

To each $(X, \omega)$, there is thus a map from $\mathrm{SL}(2, \mathbb{R})$ to $\mathcal{M}_{g}$, given by sending a group element to the equivalence class of the corresponding orbit elements. Rotations act so as to leave the Riemann surface $X$ fixed, there is thus an induced a map from $\mathrm{SO}_{2}(\mathbb{R}) \backslash \mathrm{SL}(2, \mathbb{R})$ to $\mathcal{M}_{g}$. With respect to the Teichmüller metric on $\mathcal{M}_{g}$, this map turns out to be an isometric immersion of the hyperbolic plane $\mathbb{H}^{2}=\mathrm{SL}(2, \mathbb{R}) / \mathrm{SO}_{2}(\mathbb{R})$. The resulting image is called the Teichmüller complex geodesic determined by $(X, \omega)$. We denote this by $\mathcal{G}(X, \omega)$.

The $\operatorname{SL}(2, \mathbb{R})$-stabilizer of $(X, \omega)$ induces a subgroup of the oriented isometry group of the hyperbolic plane. This induced group is the Veech group, denoted

Received by the editors July 29, 2004 and, in revised form, November 11, 2005.

2000 Mathematics Subject Classification. Primary 30F35, 11J70.

(C)2006 American Mathematical Society 
$\operatorname{PSL}(X, \omega)$. The Teichmüller complex geodesic is thus the image of the hyperbolic surface (or possibly orbifold) uniformized by the Veech group; the map between them is generically injective. We obtain a strengthening of the main result of HS3]:

Theorem 1. For any genus $g \geq 4$, there exists a complex Teichmüller geodesic in the Riemann moduli space $\mathcal{M}_{g}$ that has infinitely many cusps and infinitely many infinite ends.

We recall the definition and basic classification of ends of a hyperbolic surface below. Informally, an end is a direction in which geodesics can leave compact subsets. Finite volume hyperbolic surfaces can only have cusps as ends; briefly, a cusp, or puncture, corresponds to a conformal punctured unit disk in the surface (which cannot be completed in the surface). An end of a hyperbolic surface which corresponds to a conformal non-trivial annulus is called a hole. Holes and cusps are called finite ends, any other end is called an infinite end.

We prove our main results using the approach of translation surfaces. In particular, in terms that we make precise below, whereas cusps always form a discrete set, we give examples such that each of infinitely many directions giving an infinite end is the limit of directions of inequivalent infinite ends. A translation surface $(Y, \alpha)$ is called a Veech surface if $\operatorname{PSL}(Y, \alpha)$ is a lattice in $\operatorname{PSL}(2, \mathbb{R})$; the definition of a non-periodic connection point can be found in Subsection 2.3 .

Theorem 2. Let $(X, \omega)$ be a translation surface which is a balanced covering of $(Y, \alpha ; p)$, with $p$ a non-periodic connection point of the Veech surface $(Y, \alpha)$. Then $\operatorname{PSL}(X, \omega) \backslash \mathbb{H}^{2}$ has infinitely many cusps and infinitely many infinite ends. There is an infinite set $\Xi$ of these infinite ends such that any corresponding direction on $(X, \omega)$ is the limit of directions for distinct infinite ends.

That $\operatorname{PSL}(X, \omega)$, for $(X, \omega)$ as in Theorem 2, is infinitely generated is the main result of HS3]. For the $(X, \omega)$ of Theorem 2, the complicated geometry at infinity of $\operatorname{PSL}(X, \omega) \backslash \mathbb{H}^{2}$ is projected to a small locus of the Riemann moduli space. Restricting yet further the branch locus, we have explicit results on the geometry; the first part of this also follows from [EMM]. Here, $\Xi$ is the set of infinite ends lying above cusps of the complex Teichmüller geodesic of $(Y, \alpha)$.

Theorem 3. With notation and hypotheses as above, suppose further that $p$ is the sole branch point of the underlying cover $X \rightarrow Y$. If $X$ is of genus $g$, then (i) the complex Teichmüller geodesic generated by $(X, \omega)$ is dense in a complex algebraic surface $\mathcal{S} \subset \mathcal{M}_{g}$; and, (ii) each cusp lies within a two real-dimensional set, each infinite end in $\Xi$ lies densely in a three real-dimensional set of $\mathcal{S}$.

1.1. Outline. We prove Theorem 1 by restricting to the case where $(X, \omega)$ satisfies the hypotheses of Theorem 2 -an application of the Riemann-Hurwitz formula shows that we may assume that the corresponding $(Y, \alpha)$ be of genus two, we gave explicit examples of such $(Y, \alpha)$ which admit non-periodic connection points in [HS3], it is now known [C], Mc1] that the set of such examples is large - the existence of infinitely many cusps is proven in $\$ 4$ the existence of infinitely many infinite ends is proven in $\$ 5$, that every direction of an infinite end is the limit of inequivalent infinite end directions is also proven in \$5 discusses background for factoring projections to $\mathcal{M}_{g}$ through tori. We prove Theorem 3 in $\$ 7$. The bulk of this paper uses methods of flat geometry; this final proof relies on slightly 
different ideas. Therefore, in $\$ 7.1$ we sketch the proof, giving background material both there and at the beginning of each of the following subsections. In $\S 2$ we give background material on translation surfaces and certain $\mathrm{SL}(2, \mathbb{R})$-actions, in $\S 3$ we discuss projections of horocycles to the moduli space $\mathcal{M}_{g}$. The main results rest upon the elementary tools presented in $\S 4$.

1.2. Comments on a related setting. That a hyperbolic surface uniformized by an infinitely generated Fuchsian group of the first kind admits infinite ends can be deduced already from $[\mathrm{Fo}$, Theorem 17. Thus, the examples of infinitely generated Veech group given by McMullen Mc2 must also have infinite ends. Each of McMullen's examples has its associated Teichmüller complex geodesics lying densely on a symmetric Hilbert modular surface, all ends must lie in the finitely many cusps of the Hilbert modular surface.

1.3. Comments on a related paper. Eskin, Marklof, and Morris have recently completed [EMM], a work on flows on moduli spaces of translation surfaces which are branched covers of Veech surfaces. Although they consider branching of the type we address here, they do not explore the geometry of ends of these Teichmüller geodesics of the branched covers. However, they do discuss $\mathrm{SL}(2, \mathbb{R})$-orbits of branched covers and the location of branch loci. In particular, results such as part (i) of Theorem 3 can be deduced from their work.

\section{ACKNOWLEDGMENT}

It is a pleasure to thank F. Dal'bo, G. Forni, and J. Smillie for helpful discussions. In particular, we thank G. Forni for his ideas which are reflected in 6 . We thank A. Eskin for continued interest in these matters. It is also a pleasure to acknowledge various helpful suggestions from the referee.

\section{BACKGRound: SL(2, R)-ACTION ON TRANSLATION SURFACES}

In order to establish notation, we review basic notions. See [MT for more details; [HS1, HS2 and HS3] use related notation and notions. See also Mc1, [Mc2], Mc3], and [Mc4] for recent related developments.

2.1. Translation surfaces and affine diffeomorphisms. A translation surface is a real surface with an atlas such that all transition functions are translations. As usual, we consider maximal atlases. Given a Riemann surface $X$ and a holomorphic 1-form $\omega \in \Omega(X)$, integration of $\omega$ induces local coordinates on $X$ away from the zeros of $\omega$; regarding $\mathbb{C}$ with its standard structure as $\mathbb{R}^{2}$, transition functions are easily seen to be translations. In particular, directions of flow on a translation surface are well defined by pull-back from the plane. One easily checks that the translation surface associated to $(X, \omega)$ is well defined up to isomorphism of translation surface (such isomorphism is realized by local pure translations); see Section 2.1 of $\mathrm{HS} 3$ for a more formal discussion.

The holomorphic 1-form $\omega$ induces a flat metric on $X$ off of its zeros; when the metric is completed, a zero of multiplicity $k$ gives a conical singular point, of cone angle $2(k+1) \pi$. We consider only translation surfaces of this type; the notation $(X, \omega)$ hence denotes both the ordered pair of Riemann surface $X$ with holomorphic 1 -form $\omega$, and the translation surface structure, with which $\omega$ equips $X$. 
Given a translation surface $(X, \omega)$, and an element $A \in \mathrm{SL}(2, \mathbb{R})$, we can postcompose the coordinate functions of the charts of the atlas of $(X, \omega)$ by $A$. It is easily seen that this again gives a translation surface, denoted by $A \circ(X, \omega)$.

Given a translation surface $(X, \omega)$, let $X^{\prime}$ denote the surface which arises from deleting the singularities of $(X, \omega)$. An affine diffeomorphism $f$ of $(X, \omega)$ is a homeomorphism of $X$ that restricts to a diffeomorphism of $X^{\prime}$ whose differential at all points is a constant element of $\operatorname{SL}(2, \mathbb{R})$. In other terms, $f$ induces a realaffine map in the charts of the $\omega$-atlas on $X$, of constant (Jacobian) derivative. The orientation-preserving affine diffeomorphisms form a group, denoted $\operatorname{Aff}(X, \omega)$. The subgroup of $\mathrm{SL}(2, \mathbb{R})$ which arises as the derivatives of affine diffeomorphisms is denoted by $\operatorname{SL}(X, \omega)$.

If $\phi, \psi \in \operatorname{Aff}(X, \omega)$ have the same derivative, then the diffeomorphism $\phi \circ \psi^{-1}$ acts by pure translations. This diffeomorphism thus preserves the conformal structure on the underlying translation surface; that is, $\phi \circ \psi^{-1}$ is a conformal automorphism of the Riemann surface $X$ (that preserves $\omega$ ). The kernel of the group homomorphism from $\operatorname{Aff}(X, \omega)$ to $\operatorname{SL}(X, \omega)$ is hence certainly finite whenever $X$ is of genus greater than one. By pull-back on charts, each $\phi \in \operatorname{Aff}(X, \omega)$ also gives a translation structure on the underlying surface. Indeed, if $A$ is the constant derivative of $\phi$ with respect to the $\omega$-charts, then $\phi$ is an isomorphism of the translation surface $A \circ(X, \omega)$ with $(X, \omega)$. Thus, the $\operatorname{SL}(2, \mathbb{R})$-stabilizer of $(X, \omega)$ is $\operatorname{SL}(X, \omega)$.

2.2. Teichmüller complex geodesic, Veech group. Fix some $(X, \omega)$, and let $F: \operatorname{SL}(2, \mathbb{R}) \rightarrow \Omega \mathcal{M}_{g}$ be given by $A \mapsto A \circ(X, \omega)$; let $\pi: \Omega \mathcal{M}_{g} \rightarrow \mathcal{M}_{g}$ denote the standard projection. Note that the underlying Riemann surface $Y$ is common to the $\mathrm{SO}_{2}(\mathbb{R})$-orbit of any $(Y, \alpha)$ - the image of $(Y, \alpha)$ under a rotation is $(Y, \zeta \cdot \alpha)$, where $\zeta \in S^{1}$ is the complex number which induces the rotation. Thus, $\pi \circ F$ induces a map (from right cosets) $f_{\omega}: \mathrm{SO}_{2}(\mathbb{R}) \backslash \mathrm{SL}(2, \mathbb{R}) \rightarrow \mathcal{M}_{g}$. The image of $f_{\omega}$ is $\mathcal{G}(X, \omega)$, the Teichmüller complex geodesic associated to $(X, \omega)$.

Right multiplication gives the natural right group action of $\mathrm{SL}(2, \mathbb{R})$ on the cosets $\mathrm{SO}_{2}(\mathbb{R}) \backslash \mathrm{SL}(2, \mathbb{R})$. The center, $\{-I, I\}=\langle-I\rangle$, of $\mathrm{SL}(2, \mathbb{R})$ is a subgroup of $\mathrm{SO}_{2}(\mathbb{R})$, and one finds that $\operatorname{PSL}(2, \mathbb{R})=\mathrm{SL}(2, \mathbb{R}) /\langle-I\rangle$ acts faithfully. The $\operatorname{PSL}(2, \mathbb{R})$-stabilizer of the map $f_{\omega}$ is the Veech group $\operatorname{PSL}(X, \omega)$, the image in $\operatorname{PSL}(2, \mathbb{R})$ of $\mathrm{SL}(X, \omega)$. This is trivially verified: $f\left(\mathrm{SO}_{2}(\mathbb{R}) \cdot A\right)=f\left(\mathrm{SO}_{2}(\mathbb{R}) \cdot B\right)$ implies that there is some rotation $R$ such that $B^{-1} R A \in \operatorname{SL}(X, \omega)$. Thus, the class $\mathrm{SO}_{2}(\mathbb{R}) \cdot A$ is in the $\operatorname{PSL}(X, \omega)$-orbit of $\mathrm{SO}_{2}(\mathbb{R}) . B$. That $f_{\omega}$ is single-valued on each such orbit is obvious. The map $f_{\omega}$ is generically injective; it can only fail to be injective at some $(Y, \alpha)$ if $\operatorname{Aut}(Y)$ is larger than the automorphism group of Riemann surfaces corresponding to neighboring points of $\mathcal{M}_{g}$; see $[\mathrm{R}]$ and $[\mathrm{P}]$. When the Veech group uniformizes a true surface, then $f_{\omega}$ gives a ramified cover normalizing, in the sense of algebraic curves, $\mathcal{G}(X, \omega)$.

In Vch2, Veech proved that $\operatorname{PSL}(X, \omega)$ is a discrete subgroup of $\operatorname{PSL}(2, \mathbb{R})$. That is, it is a Fuchsian group. The Veech Dichotomy, also proven in [Vch2], states that if $\operatorname{PSL}(X, \omega)$ is a lattice - that is, if $\operatorname{PSL}(X, \omega) \backslash \mathbb{H}^{2}$ has finite hyperbolic area, then the linear flow in any direction of the surface $(X, \omega)$ is either ergodic or periodic. Thus, one says that a translation surface is a Veech surface if $\operatorname{PSL}(X, \omega)$ is a lattice.

2.3. Saddle connections, parabolic directions, and special points. Any translation surface of genus greater than one must have singularities. A geodesic 
line emanating from a singularity is called a separatrix; a separatrix connecting singularities (with no singularity on its interior) is a saddle connection. We call a non-singular $p \in(X, \omega)$ a connection point if every separatrix passing through $p$ extends to a saddle connection.

Given a direction on a translation surface, there is a corresponding point of $\partial \mathbb{H}^{2}$ : Expressing the direction as a slope $\theta$, the point on $\partial \mathbb{H}^{2}$ is $1 / \theta$; this is due to the fact that $\operatorname{SL}(2, \mathbb{R})$ acts on $(X, \omega)$ by way of its linear action on $\mathbb{R}^{2}$, but the $\operatorname{SL}(2, \mathbb{R})$ action on $\mathbb{H}^{2}$ is fractional linear. We say $\theta$ is a parabolic direction for $(X, \omega)$ if there is a parabolic element of $\operatorname{PSL}(X, \omega)$ whose associated affine diffeomorphism preserves the direction $\theta$. (In fact, the full Veech Dichotomy states that the nonergodic directions of a Veech surface are parabolic.) We also call these associated affine diffeomorphisms parabolic. Thus for example, an element of $\mathrm{SL}(2, \mathbb{R})$ arising from an element of $\operatorname{Aff}(X, \omega)$ which fixes the vertical direction (should such an element exist) acts on the extended hyperbolic plane so as to fix the boundary point $\xi=0$.

A point $p \in(X, \omega)$ of finite orbit under the group of affine diffeomorphisms is called a periodic point. A point of finite orbit under the subgroup of affine diffeomorphisms generated by the two (primitive) parabolic diffeomorphisms for some pair of transverse parabolic directions is called a rational point for $(X, \omega)$. In GHS, one shows that non-periodic points have dense orbits. In HS3, we show that every connection point on a Veech surface is a rational point.

2.4. Infinitely generated groups using ramified coverings. To date, constructions of infinitely generated Veech groups rely on the following basic fact of Fuchsian groups: (1) a Fuchsian group of the first kind (that is, whose limit set is all of $\partial \mathbb{H}^{2}$ ) is either a lattice group or else is infinitely generated. In HS3, we used ramified coverings of Riemann surfaces. Given a ramified covering $f: X \rightarrow Y$ of Riemann surfaces, let $\operatorname{Br}(f) \subset Y$ be the branch locus of $f$ and $\operatorname{PSL}(Y, \alpha ; \operatorname{Br}(f))$ the image in $\operatorname{PSL}(Y, \alpha)$ of the affine diffeomorphisms which stabilize $\operatorname{Br}(f)$. If every point of the fiber above each non-singular branch point is a point of ramification, then the translation surface $\left(X, f^{*} \alpha\right)$ is a balanced translation covering - see HS2. for the definition of this term introduced by E. Gutkin - of the marked translation surface $(Y, \alpha ; \operatorname{Br}(f))$; Veech groups of translation surfaces related by a balanced covering are commensurable [Vo, GJ]. From this, $(2) \operatorname{PSL}\left(X, f^{*} \alpha\right)$ is commensurable to $\operatorname{PSL}(Y, \alpha ; \operatorname{Br}(f))$; and, (3) if there is an element of the branch locus that is non-periodic (that is, such that its $\operatorname{Aff}(Y, \alpha)$-orbit is of infinite order), then $\operatorname{PSL}(Y, \alpha ; \operatorname{Br}(f))$ is of infinite index in $\operatorname{PSL}(Y, \alpha)$. When (2) and (3) hold, we easily conclude that $\operatorname{PSL}\left(X, f^{*} \alpha\right)$ fails to be a lattice.

Given any point of a translation surface, the directions of separatrices through the point are dense (see, Lemma 1 of [HS3], in turn essentially a result of [Vo]); the direction of any saddle connection on a Veech surface is in fact a parabolic direction. One finds that if the branch locus contains a connection point, then the parabolic directions of $\operatorname{PSL}(Y, \alpha ; \operatorname{Br}(f))$ are dense in $\partial \mathbb{H}^{2}$, and thus the limit set of this group is certainly all of $\partial \mathbb{H}^{2}$. Now, if the connection point is non-periodic, then we conclude that $\operatorname{PSL}(Y, \alpha ; \operatorname{Br}(f))$ is infinitely generated; again, the commensurable $\operatorname{PSL}\left(X, f^{*} \alpha\right)$ is therefore also infinitely generated. But, we show in HS3 that there exist $(Y, \alpha)$ that admit non-periodic connection points, and thus prove the existence of infinitely generated $\operatorname{PSL}(X, \omega)$. 
For an explicit example, we showed in HS3 (see in particular Proposition 7 there) that the genus two surface given by the golden cross can be taken to play the role of $(Y, \alpha)$. This surface has exactly one singularity, say $s$; let us also choose one of its infinitely many non-periodic connection points, $p$. If $g=2 d$ is even, then we can take $d$ copies of our genus two surface, with a slit from $s$ to $p$; identify these with the usual process after having chosen some transitive permutation on $d$ letters. This gives a degree $d$ ramified covering, whose branch locus consists of $\{s, p\}$, and which is totally ramified above each of these points. The Riemann-Hurwitz formula then shows that the covering Riemann surface does have genus $g$. One can choose the odd genus $g=3+2 k$ by way of double coverings: Choose $4 k$ connection points of our genus two surface; create $2 k$ slits and glue two copies in the standard manner. For more on creating ramified coverings, see [D].

2.5. Fuchsian groups and ends. A complex Teichmüller geodesic is 'morally' the same as the quotient of the hyperbolic plane by the corresponding Veech group. In order to address phenomena such as ends, we briefly discuss related notions in the setting of Fuchsian groups.

Definition 1. The (open) horodisk of Euclidean radius $r$ based at finite $\xi \in \partial \mathbb{H}^{2}$ is the Euclidean disk of radius $r$ in $\mathbb{H}^{2}$ that is tangent to $\partial \mathbb{H}^{2}$ at $\xi$. We also refer to the half-plane above $y=r$ as the horodisk of radius $r$ based at $\xi=\infty$.

Of the following, only the last is a new term.

Definition 2. Let $\Gamma$ be a Fuchsian group.

(1) A Dirichlet region for a Fuchsian group $\Gamma$ associated to a point $z \in \mathbb{H}^{2}$ is the set of all closest $\Gamma$-orbit representatives to $z$.

(2) A Dirichlet point for a Fuchsian group $\Gamma$ is a point $\xi \in \mathbb{H}^{2} \cup \partial \mathbb{H}^{2}$ which is on the boundary of some Dirichlet region.

(3) A parabolic point is a fixed point of a parabolic element of $\Gamma$.

(4) A cusp for $\Gamma$ is the $\Gamma$-equivalence class of a parabolic point of $\Gamma$.

(5) A fat point for $\Gamma$ is a point $\xi \in \partial \mathbb{H}^{2}$ such that there is a fundamental domain for $\Gamma$ that includes a horodisk based at $\xi$.

Remark 1. We were surprised to be unable to find in the literature a term for what we have dubbed a 'fat point'.

An end of a manifold $\mathcal{S}$ is determined by a cover of $\mathcal{S}$ by compact sets $K_{i}$, with each $K_{i}$ contained in the interior of $K_{i+1}$; an end $\mathcal{E}$ is a sequence of components $\mathcal{E}_{i}$ in the complement of $K_{i}$ such that $\mathcal{E}_{i+1} \subset \mathcal{E}_{i}$. The definition of an end is independent of choice of sequence $K_{i}$; see [Th]. An end $\mathcal{E}$ of a hyperbolic surface is called a cusp if for all sufficiently large $i, \mathcal{E}_{i}$ is conformally equivalent to the complex punctured unit disk. An end is called a hole if for all sufficiently large $i, \mathcal{E}_{i}$ is conformally equivalent to a complex annulus. These two types of ends are called finite, an infinite end is an end which is not finite. See [Ha] for discussion of the geometry of various types of ends of a hyperbolic surface. As explained in 92.4 , the hyperbolic surfaces that we address here are of the first kind, any infinite end that we discuss will be of the type called by Haas an infinite end of the first kind. (Note that each of the above definitions continues to be valid upon allowing $\mathcal{S}$ to be a hyperbolic orbifold, although there is then also the further possibility that the set of elliptic fixed points enters arbitrarily far into an end.) 
If a Fuchsian group is of the second kind, then it may have fat points inside an interval contained in the complement of its limit set. Such an interval corresponds to a hole in the corresponding surface. However, if the group is of the first kind, then there are no such intervals.

Lemma 1. If $\xi$ is a fat point for a Fuchsian group of the first kind $\Gamma$, then the $\Gamma$-equivalence class of $\xi$ corresponds to an infinite end of $\Gamma \backslash \mathbb{H}^{2}$.

The following result is well known.

Lemma 2. Finite degree ramified coverings of hyperbolic surfaces or orbifolds map: finite ends to finite ends and infinite ends to infinite ends.

Remark 2. In \$5, we identify infinite ends by way of fat points for a certain group, say $\Gamma$. These fat points are parabolic points for a related, larger group, say $G$. Now, if $G$ is any Fuchsian group, and $\xi$ is a parabolic point for $G$, then for any subgroup $\Gamma \subset G$ admitting a fundamental domain which contains some horodisk based at $\xi$, one can easily show that there is some $z \in \mathbb{H}^{2}$ such that $\xi$ is a Dirichlet point for $\Gamma$ relative to $z$.

Nicholls showed, see $\mathbb{N} 1, \mathbb{N 2}$, that a Dirichlet point $\xi \in \partial \mathbb{H}^{2}$ for some $\Gamma$ is either a parabolic fixed point, or is such that there exists $a \in \mathbb{H}^{2}$ whose $\Gamma$-orbit meets the closure of some horodisk based at $\xi$ but such that this orbit does not meet the (open) horodisk itself. Recall that $\xi \in \partial \mathbb{H}^{2}$ is called, after Sullivan [S], a Garnett point for $\Gamma$ relative to $a$ if the largest horodisk based at $\xi$ containing no element of the $\Gamma$-orbit of $a$, has its closure also not meeting this orbit. A point $\xi$ is a rigid Garnett point for $\Gamma$ if $\xi$ is Garnett with respect to all $a \in \mathbb{H}^{2}$. From the Nicholls result, the fat points we use are not rigid Garnett points. We do not yet know if they can in fact be Garnett points.

2.6. Cylinders and horocyclic flow. A cylinder on a translation surface $(X, \omega)$ is a maximal connected set of homotopic simple closed geodesics. If the genus of $X$ is greater than one, then every cylinder is bounded by saddle connections. Upon cutting the cylinder along a line perpendicular to the direction of flow, we can represent it as indicated in Figure 1] of width $w$ and height (or circumference) $h$. A cylinder has its affine Dehn twist $(x, y) \mapsto(x, y+x h / w \bmod \mathbb{Z} h)$. The modulus of the cylinder is $\mu=w / h$. If for some direction $\theta$ the surface $(X, \omega)$ decomposes into cylinders in this direction, then we say that $\theta$ is a (completely) periodic direction. Veech [Vch2] shows that a completely periodic direction $\theta$ is a parabolic direction if and only if the various cylinders in the direction $\theta$ have commensurable inverse moduli: all $h_{i} / w_{i} \cdot w_{j} / h_{j}$ are rational.

The Teichmüller horocyclic flow is given by $h_{s} \circ(X, \omega)$, where $h_{s}:=\left(\begin{array}{ll}1 & 0 \\ s & 1\end{array}\right)$, with $s \in \mathbb{R}$. The orbit of $(X, \omega)$ under this flow is called a horocycle.

Suppose that $(X, \omega)$ is completely periodic in the vertical direction, that is $(X, \omega)$ has a decomposition into vertical cylinders. The effect of $h_{s}$, with $s>0$, restricted to a cylinder of the decomposition is to change the relative positions of the singularities on the right-hand side of the cylinder with respect to those on the left. It is easily seen that on a given vertical cylinder of height $h$ and width $w$, that a standard affine twist on the cylinder has been performed when $s=h / w$. (Thus, when $s$ is the multiplicative inverse of the modulus of the cylinder.) See Figure 1 .

If the various moduli of the cylinders are commensurable, then there is a least positive $s$ such that $h_{s}$ induces on each cylinder an integer power of the basic Dehn 

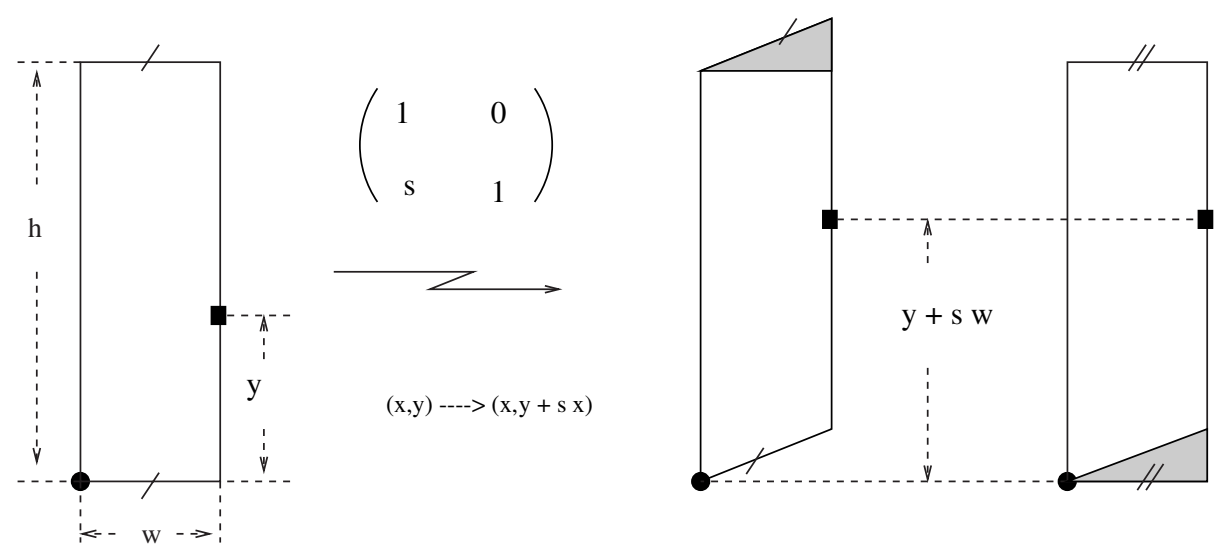

FiguRe 1. Horocyclic flow: Moving a singularity

twist of the cylinder. This $h_{s}$ is then easily seen to belong to $\operatorname{SL}(X, \omega)$. Indeed, the element $h_{s}$ then generates a parabolic subgroup of $\operatorname{PSL}(X, \omega)$, and the $\operatorname{PSL}(X, \omega)$ conjugacy class of this parabolic subgroup corresponds to a cusp.

2.7. Geodesic flow, horodisks and fat directions. Teichmüller geodesic flow is given by $g_{t} \circ(X, \omega)$, where $g_{t}=\left(\begin{array}{cc}e^{t / 2} & 0 \\ 0 & e^{-t / 2}\end{array}\right)$, with $t \in \mathbb{R}$. For positive $t$, the effect of $g_{t}$ restricted to a vertical cylinder decomposition is to decrease heights and increase widths. Note that vertical saddle connection lengths tend to zero as $t \rightarrow \infty$. Thus, if $(X, \omega)$ has saddle connections in the vertical direction, then for $t$ sufficiently large, the shortest saddle connections for $g_{t} \circ(X, \omega)$ are in the vertical direction.

Given a direction $\theta$, let $R_{\theta}$ denote the element of $\mathrm{SO}(2, \mathbb{R})$ that rotates the plane so as to send the vertical direction to $\theta$. A horodisk of index $T$ corresponding to a direction $\theta$ of $(X, \omega)$ is the union over $t>T$ of all the horocycles $h_{s}\left(g_{t} R_{\theta} \circ(X, \omega)\right)$.

Definition 3. A direction $\theta$ of $(X, \omega)$ is a fat direction if there is a positive real $T$ such that no two points of the horodisk of index $T$ corresponding to $\theta$ are $\operatorname{SL}(X, \omega)$ equivalent.

The following is immediate.

Lemma 3. Let $(X, \omega) \in \Omega \mathcal{M}_{g}$, and let $\theta \in \mathbb{P}^{1}(\mathbb{R})$ be a direction for $(X, \omega)$. Let $\xi \in \partial \mathbb{H}^{2}$ be given by $\xi=1 / \theta$. If $\theta$ is a parabolic direction, then $\xi$ is a parabolic point of $\operatorname{PSL}(X, \omega)$; if $\theta$ is a fat direction, then $\xi$ is a fat point for $\operatorname{PSL}(X, \omega)$.

\section{Elementary tools}

In HS2, we studied an invariant of the geometry at a cusp of a Teichmüller complex geodesic - the multiplicities and relative lengths of saddle connection vectors. In a similar manner, we use the following elementary notion to differentiate amongst infinite ends.

Definition 4. If $p$ lies interior to the sides of an oriented cylinder, then the splitting ratio of $p$ is the ratio of the distance from the left-hand side of the cylinder to $p$ to the width of the cylinder. (See Figure 2 .) 


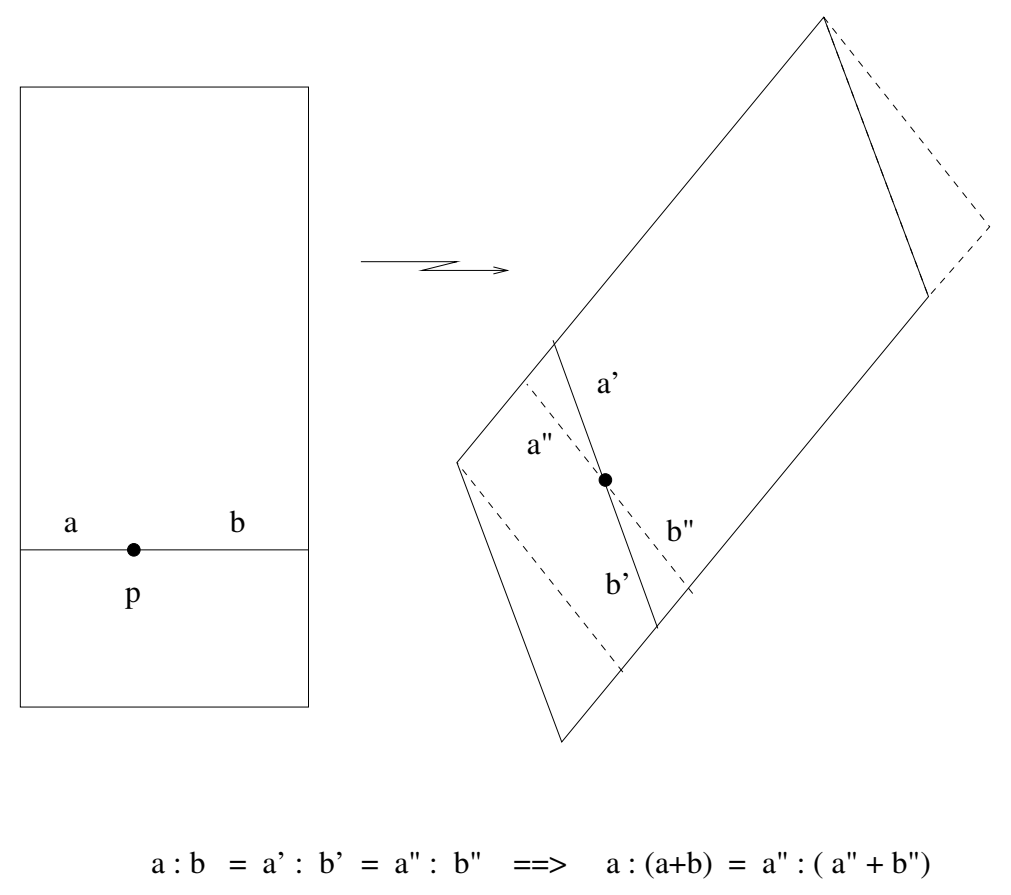

FIGURE 2. Preserving splitting ratios

The following easy result is crucial to our treatment of infinite ends. See Lemma 4 of [HS1 and Proposition 3 of [HS3 for similar use of cylinder splittings. Recall that given $p$ on a translation surface $(Y, \alpha)$, we let $\operatorname{PSL}(Y, \omega ; p)$ denote the subgroup of $\operatorname{PSL}(Y, \alpha)$ arising from the stabilizer of $p$ in $\operatorname{Aff}(Y, \alpha)$.

Proposition 1. Let $(Y, \alpha)$ be a translation surface. If $\xi \in \partial \mathbb{H}^{2}$ is a parabolic point of $\operatorname{PSL}(Y, \alpha)$ and $p \in Y$ has irrational splitting ratio for some cylinder of the cylinder decomposition in the corresponding parabolic direction, then $\xi$ is a fat point for $P S L(Y, \alpha ; p)$.

Proof. Since $\xi$ is a parabolic point, there is indeed a cylinder decomposition in the corresponding direction. Furthermore, the elements of $\operatorname{PSL}(Y, \alpha)$ that fix $\xi$ are exactly the (globally constant) derivatives of the parabolic affine diffeomorphisms that fix the corresponding direction. The point $p$ irrationally splits one of these cylinders, hence no power of the fundamental affine Dehn twist of this cylinder can fix $p$. However, every affine diffeomorphism that preserves the vertical restricts to this cylinder to be some power of this Dehn twist. Thus, none of the parabolic elements of $\operatorname{PSL}(Y, \alpha)$ for this direction are in $\operatorname{PSL}(Y, \omega ; p)$. Since these parabolic elements form a single cyclic subgroup, distinct elements of this type define distinct $\operatorname{PSL}(Y, \alpha ; p)$-cosets.

We now choose a fundamental domain for $\operatorname{PSL}(Y, \alpha)$ with the parabolic point $\xi$ on its boundary. There is some horodisk based at $\xi$ which meets this fundamental domain in a triangle, with one vertex at $\xi$. But, a fundamental domain for $\operatorname{PSL}(Y, \alpha ; p)$ can be given as the union over the $\operatorname{PSL}(Y, \alpha ; p)$-cosets of the images of our chosen fundamental domain by coset representatives. Since the chosen horodisk 
based at $\xi$ is filled out by the image of the intersection of our fundamental domain by the parabolic elements of $\operatorname{PSL}(Y, \alpha)$ fixing $\xi$, we find that $\xi$ is indeed a fat point of $\operatorname{PSL}(Y, \alpha ; p)$.

Lemma 4. Splitting ratios are $S L(2, \mathbb{R})$ invariant.

Proof. Let $(X, \omega)$ be a translation surface, and suppose that $p \in X$ splits some cylinder with ratio $\theta$. For ease of discussion, we may and do assume that the cylinder, say $\mathcal{C}$, is a vertical cylinder. Suppose that $A \in \mathrm{SL}(2, \mathbb{R})$.

We develop $\mathcal{C}$ to $\mathbb{R}^{2}$ in the standard fashion. The ratio $\theta$ is the ratio of the length of the horizontal line segment passing from the left-hand side of $\mathcal{C}$ to $p$ to the width of the cylinder. Of course, this second length is the length of the horizontal line segment joining the sides of $\mathcal{C}$ and passing through $p$.

The image of the cylinder by $A$ is again a cylinder, say $\mathcal{D}$, with obvious development. The horizontal line segment crossing $\mathcal{C}$ upon which $p$ lies has as its image some segment in $\mathcal{D}$; since $A$ acts linearly, the ratio of the length of the initial segment of this image - from the appropriate side of the cylinder to the image of $p$ - to the length of the full image segment is again $\theta$.

Draw the line segment perpendicular to the sides of the cylinder which passes through the image of $p$. Due to the equality of the opposite angles so defined, ratios of corresponding sides of the triangles so formed are equal. A trivial cancellation, see Figure 2, shows that the splitting ratio is indeed preserved.

We have the immediate consequence.

Corollary 1. Affine diffeomorphisms preserve splitting ratios of cylinders.

Lemma 5. Suppose that $\mathcal{C}$ and $\mathcal{D}$ are cylinders lying in transverse directions which have non-trivial intersection. If $p$ irrationally splits $\mathcal{C}$, then the orbit of $p$ under the affine Dehn twist of $\mathcal{C}$ meets the cylinder $\mathcal{D}$ so as to give infinitely many distinct irrational splitting ratios with respect to $\mathcal{D}$.

Proof. Let $\theta$ denote the splitting ratio of $p$ with respect to $\mathcal{C}$. By Lemma 4 , we may normalize such that $\mathcal{C}$ is in the vertical direction and $\mathcal{D}$ is in the horizontal direction. Let $\mathcal{R}$ be a rectangle in which our two cylinders meet. Let us develop the cylinders such that $\mathcal{R}$ is bounded below by the line $y=y_{0}$ and above by $y=y_{0}+\lambda$. Let us denote by $h$ the vertical height of $\mathcal{C}$. Thus in these coordinates, the orbit of $p$ by the vertical Dehn twist has heights $n \theta h \bmod \mathbb{Z} h$, with $n \in \mathbb{N}$. But, this is exactly the set of $\{n \theta\} h$, where $\{x\}$ denotes the fractional part of the real number $x$.

It suffices for us to show that there are infinitely many distinct ratios $(\{n \theta\} h) / \lambda$ which are irrational, and lie between $y_{0}$ and $y_{0}+\lambda$. For completeness' sake, we show this elementary, presumably well known, result.

Let $\nu:=\lambda / h$. Now, if $\{n \theta\}$ is a rational multiple of $\nu$, then it is necessary that $\theta=\frac{a \nu+b}{c}$ for $a, b, c$ integers, with $a, c$ non-zero.

Suppose now that $\theta$ is of the form $\frac{a \nu+b}{c}$, and there is an $n$ such that $\{n \theta\}=r \nu / s$ for non-zero integers $r$ and $s$. We find:

$$
n \frac{a \nu+b}{c}-k=r \nu / s, \quad \text { with } k=\lfloor n \theta\rfloor .
$$


Since $\theta$ is irrational, it is not linearly related to a rational number. Hence, in particular, we must have that $k=n b / c$. That is, $\lfloor n \theta\rfloor=n b / c$; but then $0<$ $\theta-b / c<1 / n$. This can only occur for a finite number of $n$.

Since in the worst case scenario there are only a finite number of orbit elements to avoid, and the orbit of $p$ is dense in the circle on the vertical cylinder, we conclude that there are always infinitely many elements of the orbit which irrationally split the horizontal cylinder.

The following is also an elementary tool in our approach.

Lemma 6. The affine diffeomorphisms of a translation surface preserve the set of connection points.

Proof. Suppose that $\phi \in \operatorname{Aff}(X, \omega)$ is an affine diffeomorphism and $p \in X$ is a connection point. Given a separatrix passing from a singularity to $\phi(p)$, we apply $\phi^{-1}$ to find a separatrix passing from some singularity through $p$. Since $p$ is a connection point, this separatrix extends to a saddle connection. But, the image by $\phi$ of this extended separatrix extends our initial separatrix passing through $\phi(p)$. Since $\phi$ sends saddle connections to saddle connections, we are done.

\section{INFINITELY MANY CUSPS}

We use the invariant studied in [HS2] to distinguish cusps.

Proposition 2. Let $(X, \omega)$ be a balanced cover of $(Y, \alpha ; p)$, with $p$ a non-periodic connection point of the Veech surface $(Y, \alpha)$. Then the Teichmüller complex geodesic $\mathcal{G}(X, \omega)$ has infinitely many cusps.

Proof. The translation structure of $(X, \omega)$ arises as the pull-back by a ramified holomorphic map $f: X \rightarrow Y$ of Riemann surfaces, with $\omega=f^{*} \alpha$. Recall from $\$ 2.4$ that the Veech group $\operatorname{PSL}(X, \omega)$ is commensurable to the subgroup of $\operatorname{PSL}(Y, \alpha)$ formed by the derivatives of the stabilizer in $\operatorname{Aff}(X, \alpha)$ of $p$.

By Theorem 2 of GHS, the image of the non-periodic $p$ under the action of the affine diffeomorphism group is dense on $(Y, \alpha)$. Let $l$ be the length of the shortest saddle connection of $(Y, \alpha)$. Given $\epsilon<l / 2$, we can find an image of $p$ under $\operatorname{Aff}^{+}(X, \omega)$, say $q$, which is within $\epsilon$ of one of the singularities, say $\sigma$. There is a unique separatrix of length less than $\epsilon$ connecting $q$ and $\sigma$. By Lemma 6, we can extend this separatrix to a saddle connection, passing from $\sigma$ to a singularity $\tau$. (Of course, $\tau$ may equal $\sigma$.)

Let $\phi$ be an affine diffeomorphism mapping $q$ to $p$. The image by $\phi$ of the above saddle connection is a saddle connection passing through $p$. Since $(Y, \alpha)$ is a Veech surface, the direction of any saddle connection is a parabolic direction. Since $p$ lies on this saddle connection, $p$ is preserved by the Dehn twist of either of the cylinders (in this given direction) upon which $p$ lies. Thus this direction is a parabolic direction for $(X, \omega)$.

Consider a single lift to $(X, \omega)$ of the saddle connection that we have just determined. This lift is the union of two saddle connections on $(X, \omega)$ : the first joins a lift of $\phi(\sigma)$ to a lift of $p$, the second joins the lift of $p$ to a lift of $\phi(\tau)$. (The connection vectors of these saddle connections are parallel!)

Now, the lengths of these saddle connections on $(X, \omega)$ are equal to the lengths of the corresponding segments on $(Y, \alpha)$. But, the affine diffeomorphism $\phi$ preserves ratios of segment lengths; thus the ratio of the lengths of the saddle connections on 
$(X, \omega)$ equals the ratio of the length along the separatrix from $\sigma$ to $q$ to the length along its continuation from $q$ to $\tau$. We find that this ratio is less than $\epsilon / 2 l$.

Our construction thus allows us to find pairs of parallel connection vectors, in parabolic directions, of $(X, \omega)$ that are of arbitrarily small ratio. However, the number of connection vectors in any given direction is finite. Furthermore, the ratios of the various connection vectors lying in a parabolic direction is invariant under the action of $\operatorname{SL}(X, \omega)$ - see $\mathrm{HS} 2$. We conclude that $(X, \omega)$ has infinitely many inequivalent parabolic directions. But, each of these parabolic directions determines a distinct cusp of $\operatorname{PSL}(X, \omega) \backslash \mathbb{H}^{2}$. Since the Teichmüller complex geodesic $\mathcal{G}(X, \omega)$ is a generically injective image of this quotient, it too has infinitely many cusps.

\section{INFINITELY MANY INFINITE ENDS}

We now give the proof of Theorem 2. Thus, we continue with $(X, \omega)$ as a ramified covering of $(Y, \alpha)$ as above. The periodic directions on $(X, \omega)$ are exactly those given by the periodic, and hence parabolic, directions of $(Y, \alpha)$. We show that $(X, \omega)$ has many inequivalent directions which are periodic, but not parabolic.

Proposition 3. Let $(X, \omega)$ be a balanced cover of $(Y, \alpha ; p)$, with $p$ a non-periodic connection point of the Veech surface $(Y, \alpha)$. Then the Teichmüller complex geodesic $\mathcal{G}(X, \omega)$ has infinitely many infinite ends.

Proof. There is some parabolic direction of $(Y, \alpha)$ for which $p$ irrationally splits a cylinder. Without loss of generality, we may normalize so that $p$ irrationally splits a vertical cylinder and such that the horizontal is a parabolic direction for $(Y, \alpha)$.

Consider the vertical cylinder $\mathcal{C}$ in which $p$ lies, and any horizontal cylinder $\mathcal{D}$ which meets $\mathcal{C}$. By Lemma 5 , the set of splitting ratios of $\mathcal{D}$ determined by the orbit of $p$ under the vertical affine Dehn twist includes an infinite set of irrational numbers. There is some finite power of this Dehn twist which is induced by an element $\phi$ of the affine diffeomorphism group of $(Y, \alpha)$; the orbit of $p$ under the subgroup generated by this multiply-composed Dehn twist still provides an infinite set of distinct irrational splitting ratios.

For each image $q=\phi^{n}(p)$ of this latter type, apply the corresponding inverse $\phi^{-n}$. Now, $\phi^{-n}(q)=p ; \phi^{-n}(\mathcal{D})$ is a cylinder in a corresponding parabolic direction for $(Y, \alpha)$; and, $p$ splits $\phi^{-n}(\mathcal{D})$ with the ratio that $q$ splits $\mathcal{D}$. Of course, given any direction, $p$ lies interior to at most one cylinder of the possible decomposition in that direction; thus the splitting ratio defined by $p$ in that direction is well defined. Furthermore, the splitting ratio that $p$ gives is constant on directions equivalent under the stabilizer of $p$ amongst the affine diffeomorphisms of $(Y, \alpha)$. Since the $\operatorname{Aff}(Y, \alpha)$-orbit of $p$ gives infinitely many distinct irrational splitting ratios, Corollary 1 and Proposition 1 now imply that there are infinitely many fat directions that are inequivalent under the stabilizer of $p$.

By Lemma 1 $\operatorname{PSL}(Y, \alpha ; p) \backslash \mathbb{H}^{2}$ has infinitely many infinite ends. But, again as mentioned in 92.4 . $\operatorname{PSL}(X, \omega)$ is commensurable with $\operatorname{PSL}(Y, \alpha ; p)$; hence, $\operatorname{PSL}(Y, \alpha ; p) \backslash \mathbb{H}^{2}$ and $\operatorname{PSL}(X, \omega) \backslash \mathbb{H}^{2}$ admit a common finite degree (possibly ramified) covering. By Lemma 2, PSL $(X, \omega) \backslash \mathbb{H}^{2}$ also has infinitely many infinite ends. But, the Teichmüller complex geodesic $\mathcal{G}(X, \omega)$ is a generically injective image of $\operatorname{PSL}(X, \omega) \backslash \mathbb{H}^{2}$, it too must have infinitely many infinite ends.

We now complete the proof of Theorem 2 . 
Lemma 7. With hypotheses and notation as above, let $\theta$ be a fat direction of $(X, \omega)$. Then $\theta$ is the limit of $\operatorname{PSL}(X, \omega)$-inequivalent fat directions of $(X, \omega)$.

Proof. From construction, $\theta$ is a parabolic direction for $(Y, \alpha)$ and the non-periodic connection branch point $p$ irrationally splits a cylinder $\mathcal{C}$ of $(Y, \alpha)$ in this direction. Recall that some power of the Dehn twist in this cylinder is induced by an element $\phi$ of the affine diffeomorphism group of $(Y, \alpha)$. Choose any parabolic direction for $(Y, \alpha)$ that is transverse to $\theta$. There is some cylinder $\mathcal{D}$ in this direction that meets $\mathcal{C}$. The previous proof shows that there is a subsequence of $\phi^{-n}(\mathcal{D})$ giving cylinders which are split by $p$ with distinct irrational splitting ratios. Since $\phi$ is parabolic, preserving the direction $\theta$, the direction of the cylinder $\phi^{-n}(\mathcal{D})$ tends to $\theta$ as $n$ tends to infinity. Thus, $\theta$ is the limit of these inequivalent fat directions for $(Y, \alpha ; p)$. Finally, commensurability of groups allows us to choose a subsequence of these directions consisting of inequivalent fat directions for $(X, \omega)$.

\section{FACTORING HOROCYCles Through TORI}

We discuss the effects on $\mathcal{G}(X, \omega)$ of Dehn twists associated to a cylinder decomposition of $(X, \omega)$. These Dehn twists are elements of the Teichmüller modular group, and thus preserve the complex structure on $X$. We imagine that all of the following is well known. Our understanding of this material arose out of discussion with G. Forni. In this section, we state and prove Proposition 4 and the related Lemma 10, this latter is used in the next section.

Proposition 4. Suppose that the translation surface $(X, \omega)$ has a cylinder decomposition, into $n$ cylinders, in the direction $\theta$. Let $m$ be the number of distinct commensurability classes of the inverse moduli of these cylinders. Then the projection to $\mathcal{M}_{g}$ of the horocycle $\left\{h_{s} \circ\left(R_{\theta} \circ(X, \omega)\right) \mid s \in \mathbb{R}\right\}$ factors through a dense image on a real torus of dimension $m$.

The torus invoked in the above arises from Dehn twists, a simple form of this is given in the following. The proof of necessity involves some Teichmüller theory.

Lemma 8. Suppose that the translation surface $(X, \omega)$ has a cylinder $\mathcal{C}$ in the vertical direction. Let $\sigma$ be the basic affine Dehn twist in $\mathcal{C}$, extended by the identity to all of $(X, \omega)$. Then $\sigma$ defines an element of the mapping class group, $\operatorname{Mod}(g, n)$ where $X$ is $f$ genus $g$ and $(X, \omega)$ has $n$ singular points. In particular, using $\sigma$ again to denote its class, $\pi \circ \sigma((X, \omega))=\pi((X, \omega))$, where $\pi: \Omega \mathcal{M}_{g} \rightarrow \mathcal{M}_{g}$ is the standard projection.

Proof. This follows from standard material, which we sketch. Let $X^{\prime}$ denote $X$ with the zeros of $\omega$ deleted. Then $\sigma$ is a quasi-conformal homeomorphism of $X^{\prime}$ to itself; see EG] for a particularly clear exposition of such self-maps (and their effects on Teichmüller disks). Now, by taking squares, $\Omega \mathcal{M}_{g}$ is identified with a subspace of the quadratic differentials. But, the modular $\operatorname{group} \operatorname{Mod}(g, n)$ acts biholomorphically and compatibly on both the Teichmüller space of $n$ pointed marked Riemann surfaces and the space of its quadratic differentials, see the introduction to Vch1. Furthermore, this action respects the strata of squares of holomorphic 1-forms; see, in particular, Section 2 of $\underline{\mathrm{M}}$. Thus, $\sigma((X, \omega))$ determines a holomorphic 1-form on a punctured Riemann surface $Z^{\prime}$ that is biholomorphically equivalent to $X^{\prime}$. The holomorphic structure extends over the removable singularities, and hence we may indeed consider $\sigma((X, \omega))$ as an element of $\Omega \mathcal{M}_{g}$. Furthermore, the biholomorphic 
equivalence of $X^{\prime}$ and $Z^{\prime}$ extends to a biholomorphic equivalence of $X$ and $Z$. Thus, we have the equality $\pi \circ \sigma((X, \omega))=\pi((X, \omega))$.

The following is immediate.

Lemma 9. With notation as above, horocyclic flow restricts to $\mathcal{C}$ to give a map that commutes with $\sigma$. Furthermore, if $\mathcal{C}$ is of modulus $\mu$, then modulo the cyclic group generated by $\sigma$, this restricted map is parametrized by $s \bmod \mu^{-1}$.

Definition 5. Suppose that the translation surface $(X, \omega)$ has a cylinder decomposition, into $n$ cylinders, in the vertical direction. Define the following $\mathbb{R}^{n}$-action on the underlying topological surface. Given $s=(s(1), \ldots, s(n)) \in \mathbb{R}^{n}$, in the $i$ th cylinder, apply the restriction of the horocyclic flow $h_{s(i)}$.

Note that the above $\mathbb{R}^{n}$-action commutes with the group generated by the various $\sigma_{i}$. Thus, modulo this group, the $\mathbb{R}^{n}$-action defines an $n$-torus; the horocycle $h_{s} \circ(X, \omega)$ projects to a curve on this torus. Since each $\sigma_{i}$ also commutes with the projection to $\mathcal{M}_{g}$, the projection of the horocycle factors through the image of the horocycle on this torus.

But, we can synchronize the twists for cylinders of commensurable (inverse) moduli. Let $m$ be the number of these commensurability classes; for the $j$ th class, let $\tilde{\sigma}_{j}$ be the composition of the various $\sigma_{i}^{r(i)}$ in this class, where each $r(i)$ is the (minimal) appropriate power (as in the formation of parabolic affine diffeomorphisms). Again, the projection of the horocycle factors through the torus corresponding to the group generated by the $\tilde{\sigma}_{j}$.

If our direction is parabolic and there is a single commensurability class, in which case we find that the horocycle factors through a circle, which we certainly already knew! Otherwise, the image of the diagonal $(s, \ldots, s) \in \mathbb{R}^{m}$ projects to a dense curve in the torus; that is, the image of the horocycle is dense on the $m$-torus.

Finally, all of the above goes though mutatis mutandi upon replacing the vertical by any direction of a cylinder decomposition.

Lemma 10. For positive $t$, the image under $g_{t}$ of the horocycle of Proposition 4 also has projection to $\mathcal{M}_{g}$ factoring through a dense curve on a real m-torus.

Proof. For positive $t$, the effect of $g_{t}=\left(\begin{array}{cc}e^{t / 2} & 0 \\ 0 & e^{-t / 2}\end{array}\right)$ on a vertical cylinder decomposition of $(X, \omega)$ is to preserve the number of cylinders, while decreasing heights and increasing widths. Indeed, $g_{t}$ acts so as to multiply vertical cylinder moduli by $e^{t}$. Thus, the number $m$ remains constant. The rest of the proof is clear.

\section{Covers And moduli SPACE}

7.1. Overview of proof: Density in algebraic surface. Heuristically, the density of $\mathcal{G}(X, \omega)$ in its complex algebraic surface is simply due to the fact that the branch point $p$ of $(Y, \alpha)$ has dense orbit under the group of affine diffeomorphisms. There are two main steps involved in turning this into a proof: (1) $f: X \rightarrow Y$ induces a topologically identical ramified covering for each element of the $\mathrm{SL}(2, \mathbb{R})$ orbit of $(X, \omega)$, see Lemma 13 below; but the Riemann surfaces $Z$ of genus $g$ which have a map of a given topological type (in terms of the monodromy group, see the discussion in 87.3 form an analytic subvariety of $\mathcal{M}_{g}$ - this is a consequence of Wewers' thesis [W]; see Theorem 4 below. In fact, Wewers' result is algebraic in 
nature; and since $\mathcal{G}(Y, \alpha)$ is a complex algebraic curve - as shown by [Moe2], this can be seen as resulting from Smillie's Theorem, see [Vch3], the SL(2, R)-orbit of any Veech surface is closed - it follows that $\mathcal{G}(X, \omega)$ lies in an algebraic variety. Our condition of a single point of ramification allows an easy dimension count to show that $\mathcal{G}(X, \omega)$ does indeed lie in an algebraic surface. (2) To show the density of $\mathcal{G}(X, \omega)$ there, one focuses on the branch locus of $f$ and of each of the maps for the Riemann surfaces $Z$ as above. To consider these all at once, recall that the universal curve $\mathcal{M}_{h, 1}$ is the space of Riemann surfaces of genus $h$ with a marked point. We show that the branch point $p$ gives rise to a dense subset of the subset of the universal curve lying above $\mathcal{G}(Y, \alpha)$; see Lemma 11. Wewer's Theorem can then be invoked to show the density of $\mathcal{G}(X, \omega)$ in its complex algebraic surface; see Lemma 15.

Remark 3. An advantage of using the strongly algebraic result of Wewers is that one can expect that whenever some $(X, \omega)$ occurs as a ramified cover of a Veech surface, it then will be dense in some appropriate algebraic variety, whose field of definition is given in terms of the field of definition of the Teichmüller curve associated to the Veech surface and the branch locus. Of course, the question remains whether a similar statement is true under the weaker condition that $\mathrm{SL}(X, \omega)$ is infinitely generated.

7.2. Mapping to a universal curve. With our ongoing assumptions, we have

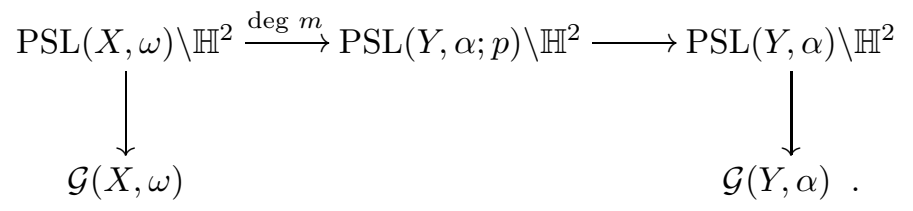

We now give what one might consider as $\mathcal{G}(Y, \alpha ; p)$, allowing us to complete the lower horizontal line to give a commutative diagram; see $\mathcal{C}^{0}$ of Lemma 12. We consider a map to a universal curve. See $[\mathrm{HM}],[\mathrm{Hu}]$, and $[\mathrm{L}$ for related ideas.

For brevity, we use the language of Teichmüller theory. The hyperbolic metric on $\operatorname{PSL}(Y, \alpha) \backslash \mathbb{H}^{2}$ allows us to identify it with the Teichmüller disk of $\alpha^{2}$ in the Teichmüller space of $X$. Let $V$ be the fiber space of corresponding marked Riemann surfaces above this disk. Note that $p$ defines a section of this fiber space, $\sigma_{p}$.

A classic result of $\mathrm{H}$. Cartan is that the quotient of a complex manifold by a properly discontinuous group of automorphisms gives a normal complex space. (A normal complex space is a complex manifold off of its singular points, and the singular locus is small in the sense that any function that is locally bounded and holomorphic on its complement extends to a holomorphic function on the whole space. [See [Na and especially $[\mathrm{Fr}$ for definitions and details.] There is also the notion of a normal algebraic variety, the standard underlying space of a normal variety over $\mathbb{C}$ is a normal complex space; see the end of Appendix VI of $[\mathrm{Fr}]$.)

Now, since the Teichmüller modular group acts properly discontinuously by biholomorphisms on Teichmüller space, one has that $\mathcal{M}_{h}$ is certainly a normal analytic space. Similarly, there is the possibility to achieve a universal marked curve over Teichmüller space, this descends to give a normal complex fiber space - the universal curve $\mathcal{M}_{h, 1}$ over $\mathcal{M}_{h}$. Here each fiber is a Riemann surface modulo its automorphism group; see, in particular, the Corollary on p. 326 of [Na]. 
Thus, $V$ projects to a normal complex fiber space $\mathcal{C}$, the restriction of the universal curve to $\mathcal{G}(Y, \alpha)$. Clearly, the section $\sigma_{p}$ projects to a subset $\mathcal{C}^{0} \subset \mathcal{C}$.

Lemma 11. Let $(Y, \alpha)$ and $p$ be as above. The projection of the fiber space $V$ to the universal curve restricted to $\mathcal{G}(Y, \alpha)$ sends the image of the section $\sigma_{p}$ to a dense subset $\mathcal{C}^{0}$.

Proof. By Theorem 2 of GHS, the non-periodic $p$ has dense $\operatorname{Aff}(Y, \alpha)$-orbit on the (marked) Riemann surface $Y$. But, $\operatorname{Aff}(Y, \alpha)$ injects into the modular group $\operatorname{Mod}(h)$, so as to give the stabilizer of the Teichmüller disk of $\alpha^{2}$. Recall that $f_{\alpha}$ is generically injective, for this argument we may assume that it is injective at the center of this disk (under its identification with $\mathbb{H}^{2}$ as above). Hence the image of $p$ under $\operatorname{Aff}(Y, \alpha)$ descends to give a dense subset of the fiber of $\mathcal{C}$ above the image of the center of the disk. Since the affine diffeomorphism group of any element in the $\mathrm{SL}(2, \mathbb{R})$-orbit of $(Y, \alpha)$ is conjugate to that of $(Y, \alpha)$, the result follows.

Lemma 12. Let $(Y, \alpha)$ and $p$ be as above. There is a generically injective analytic map $f_{p}: \operatorname{PSL}(Y, \alpha ; p) \backslash \mathbb{H}^{2} \rightarrow \mathcal{C}^{0}$.

Proof. This follows from the generic injectivity of $f_{\alpha}$ and the fact that $\operatorname{PSL}(Y, \alpha ; p)$ is exactly the group of derivatives of the affine diffeomorphisms of the translation surface $(Y, \alpha)$ that fix $p$.

In classical Teichmüller theoretic terms, the map from $\mathbb{H}^{2}$ to the image of $\sigma_{p}$ is the restriction to a certain Teichmüller disk of the standard map from $T(h, r+1)$ to $V(h, r)$; see p. 330 of $[\mathrm{Na}$.

Proposition 5. With notation as above, let $\mathcal{E}$ be an end of $P S L(Y, \alpha ; p) \backslash \mathbb{H}^{2}$. If $\mathcal{E}$ is a cusp, then the image under $f_{p}$ of $\mathcal{E}$ is homeomorphic to $\mathbb{R}^{+} \times S^{1}$. Let $\Xi$ be the set of infinite ends of $\operatorname{PSL}(Y, \alpha ; p) \backslash \mathbb{H}^{2}$ that lie above cusps of $P S L(Y, \alpha) \backslash \mathbb{H}^{2}$. If $\mathcal{E}$ belongs to $\Xi$, then the image is homeomorphic to $\mathbb{R}^{+} \times S^{1} \times A$, with $A$ a dense subset of $S^{1}$.

Proof. For $\mathcal{E}$ of either of the two types of ends considered here, let $\xi \in \partial \mathbb{H}^{2}$ correspond to $\mathcal{E}$, and let $\theta=1 / \xi$ be the corresponding direction on the translation surface $(Y, \alpha)$. Since this latter is a Veech surface, one easily shows that $\theta$ is a parabolic direction for $(Y, \alpha)$. Take a horodisk based at $\xi$ of sufficiently small radius such that it projects to the cusp of $\operatorname{PSL}(Y, \alpha) \backslash \mathbb{H}^{2}$ corresponding to $\xi$. This horodisk also projects to $\mathcal{E}$.

If $\mathcal{E}$ is a cusp, then $p$ must not irrationally split any cylinder in the $\theta$ direction. Thus $p$ is of finite order under the parabolic affine diffeomorphisms preserving the direction $\theta$. That is, $p$ is stabilized by a finite index subgroup of the subgroup of $\operatorname{Aff}(Y, \alpha)$ preserving $\theta$. Reasoning as in the proof of Lemma 10] shows that this is true upon replacing $(Y, \alpha)$ by any $g_{t} \circ(Y, \alpha)$ with $t>0$. Hence, for each point in our chosen horodisk, the horocycle based at $\xi$ passing through this point projects to a circle in both $\operatorname{PSL}(Y, \alpha ; p) \backslash \mathbb{H}^{2}$ and $\operatorname{PSL}(Y, \alpha) \backslash \mathbb{H}^{2}$. This is compatible with the projection from $\mathcal{C}$ to $\mathcal{G}(Y, \alpha)$ : The circle in $\operatorname{PSL}(Y, \alpha ; p) \backslash \mathbb{H}^{2}$ is mapped to a circle in $\mathcal{C}^{0}$ lying above a circle on $\mathcal{G}(Y, \alpha)$.

Now, suppose that $\mathcal{E}$ is an infinite end in $\Xi$. Then $p$ must irrationally split some cylinder. Consider the cylinder decomposition with this split cylinder replaced by the two newly created cylinders. This has exactly two commensurability classes of inverse moduli. Furthermore, for each point in our chosen horodisk, although the 
horocycle based at $\xi$ passing through this point projects to a circle in $\operatorname{PSL}(Y, \alpha) \backslash \mathbb{H}^{2}$, it projects to a line in $\operatorname{PSL}(Y, \alpha ; p) \backslash \mathbb{H}^{2}$. Directly related to this, the orbit of $p$ on the translation surface $(Y, \alpha)$ forms a proper dense subset of the circle in the direction $\theta$ upon which $p$ lies. The image of the line under $f_{p}$ is indeed a line lying densely in a torus contained in $\mathcal{C}$; this torus projects to the circle on $\mathcal{G}(Y, \alpha)$ arising from the horocycle. Again, the above persists under application of the Teichmüller flow, $g_{t}$, to provide a factor of $\mathbb{R}^{+}$.

7.3. Hurwitz spaces. We will show that $\mathcal{G}(X, \omega)$ lies in a complex surface of $\mathcal{M}_{g}$, related to $\mathcal{G}(Y, \alpha)$. One can discuss these matters in terms of the so-called relative Teichmüller spaces; see, for example, Remark 3 on p. 510 of [MH]. However, we use the notion of Hurwitz spaces. For this, we rely upon S. Wewers' thesis; see Moe for similar usage. This can be compared with [MSSV]'s use of the earlier results of $\mathrm{Ber}$. See also $\S 5$ of $[\mathrm{L}$ for another application of maps between moduli spaces induced by ramified covers.

We first sketch the idea. Given our $f: X \rightarrow Y$, consider the space of all ramified covers of compact Riemann surfaces $\rho: Z \rightarrow W$ where $Z$ is of genus $g$ and $W$ of genus $h$ and of the same monodromy group as $f$. By work of Wewers $W$, this space has the structure of a quasi-projective variety $\mathcal{H}$ (that is, $\mathcal{H}$ is the complement of an algebraic subvariety inside some projective variety); furthermore, there are finite degree analytic maps from $\phi: \mathcal{H} \rightarrow \mathcal{M}_{g}$ and $\Psi: \mathcal{H} \rightarrow \mathcal{M}_{h, 1}$. Viewing $\mathcal{M}_{h, 1}$ as the universal curve over $\mathcal{M}_{h}$, restrict to the algebraic (normal) surface fibering $\mathcal{C}$ over $\mathcal{G}(Y, \alpha)$. We show that $\mathcal{G}(X, \omega)$ lies densely inside the image by $\Phi$ of one of the components of $\Phi^{-1}(\mathcal{C})$.

The monodromy group of a ramified cover of topological surfaces, $\rho: A \rightarrow B$, is the permutation representation of the fundamental group of $B \backslash \operatorname{Br}(f)$ determined by fixing a non-branch point $b$ as base point and computing the action on the fiber $\rho^{-1}(b)$ of the fundamental group - each closed loop based at $b$ lifts to give paths joining elements of the fiber. Since we assume connected covers, the resulting homomorphic image of $\pi_{1}(B)$ is a transitive subgroup of the symmetric group $S_{d}$, with $d$ the degree of the cover. Deleting the branch points and their pre-images results in a true cover of surfaces; this cover is regular if the monodromy group is a normal subgroup of $S_{d}$. This cover being regular is equivalent to the ramified cover $\rho$ being Galois.

Note that change of base point results in a conjugation of monodromy group; as well, change in enumeration of the elements of the fiber $\rho^{-1}(b)$ also results in a conjugation of monodromy group. Still, it is standard practice to speak of the monodromy group of a cover, tacitly using the fact that it is rather the conjugacy class in $S_{d}$ of the group that is well defined.

The following elementary result is crucial to our discussion.

Lemma 13. Let $f:(X, \omega) \rightarrow(Y, \alpha ; \mathcal{P})$ be a balanced cover of translation surfaces, and $A \in S L(2, \mathbb{R})$. Then $f: A \circ(X, \omega) \rightarrow A \circ(Y, \alpha)$ is also a balanced cover, and has the same monodromy group.

Proof. That the $\mathrm{SL}(2, \mathbb{R})$-action preserves balanced covers is shown by both GJ] and $[\mathrm{Vo}$. Since this action does not change topology, it is clear that monodromy groups are preserved.

In roughest terms, Hurwitz spaces parametrize covers of Riemann surfaces in terms of ramification data. Wewers $[\mathrm{W}]$ obtains various results about Hurwitz 
spaces (and in particular their compactifications) by using the theory of algebraic stacks. Wewers used the notion of $G-N$ covers-appropriately defined families of covers such that all monodromy groups lie within the subgroup $N \subset S_{d}$ where $N \supset G$, and such that all geometric fibers of the family do have $G$ as monodromy group. Geometric fibers here are those elements of the family corresponding to closed points of the underlying parametrizing space - in our setting, all 'standard' points are closed points.

Recall that $\mathcal{M}_{h,[r]}$ denotes the Riemann moduli space of Riemann surfaces of genus $h$ with $r$ unordered points marked. Combining Theorem 4.1.4 and Remarks 4.1.6 and 4.1.7 of [W], with underlying ring being $\mathbb{C}$, one has the following.

Theorem 4 (Wewers). Fix a genus $h$ and a natural number $r$. Suppose that $G$ is a monodromy group realized by some cover $\rho: X \rightarrow Y$ with $Y$ of genus $h$, $\rho$ having $r$ branch points, and $X$, say, of genus $g$. Then there is a quasi-projective space $\mathcal{H}$, parametrizing all covers of genus $h$ Riemann surfaces with monodromy group $G$, with finite degree, (forgetful) algebraic maps $\Phi: \mathcal{H} \rightarrow \mathcal{M}_{g}$ and $\Psi: \mathcal{H} \rightarrow \mathcal{M}_{h,[r]}$.

7.4. Proof of Theorem 3. Suppose that $\mathcal{G}(Y, \alpha)$ is any complex Teichmüller geodesic and $\rho: X \rightarrow Y$ any cover ramified above $r$ points of $Y$. Then the restriction of the natural projection $\mathcal{M}_{h,[r]} \rightarrow \mathcal{M}_{h}$ restricts to give an $r+1$ dimensional fiber space $\mathcal{V}$ over $\mathcal{G}(Y, \alpha)$. If $\rho$ has monodromy group $G$, then $X$ corresponds to a point contained in the image of $\Phi$ applied to an appropriate component of $\Phi^{-1}(\mathcal{V})$.

Lemma 14. Let $\rho:(X, \omega) \rightarrow(Y, \alpha ; \mathcal{P})$ be a balanced cover of translation surfaces, with the corresponding cover of the underlying Riemann surfaces ramified over $r$ points. Suppose $(Y, \alpha)$ is a Veech surface.

Let $\mathcal{V}$ be the restriction to $\mathcal{G}(Y, \alpha)$ of the fiber space $\mathcal{M}_{h,[r]} \rightarrow \mathcal{M}_{h}$. Then $\mathcal{G}(X, \omega)$ is a subset of the algebraic set given by $\Phi$ applied to the pre-image under $\Psi$ of $\mathcal{V}$. In particular, $\mathcal{G}(X, \omega)$ lies within an $r+1$ complex dimensional algebraic space.

Proof. By Lemma 13 each point of $\pi(\mathrm{SL}(2, \mathbb{R}) \circ(X, \omega))$ has a ramified covering map, with the same monodromy group and number of branch points as $f$, to a Riemann surface corresponding to a point of $\mathcal{G}(Y, \alpha)$. Thus, $\mathcal{G}(X, \omega)$ is indeed contained in the image under $\Phi$ of the appropriate subset of the Hurwitz space. The fact that $\mathcal{V}$ is analytic and of dimension $r+1$ is well known; see p. 326 of [Na] and surrounding discussion. The result thus is a consequence of Wewers' Theorem 4 above.

Lemma 15. With hypotheses and notation as above, suppose further that $\mathcal{P}$ consists of a single, non-periodic connection point. Then $\mathcal{G}(X, \omega)$ lies densely on a complex algebraic surface.

Proof. We can identify $\mathcal{M}_{h,[1]}$ with $\mathcal{M}_{h, 1}$, and in fact with the universal curve over $\mathcal{M}_{h}$. Combining Lemmas 11 and 13, the single branch point of our given map $f$ and its images under the $\operatorname{SL}(2, \mathbb{R})$-action leads to a section $\sigma_{p}$ whose image is dense in the restriction of the universal curve to $\mathcal{G}(Y, \alpha)$. But, $\mathcal{G}(X, \omega)$ lies in the image by $\Phi$ of the pre-image under $\Psi$ of this dense set.

Now, since the Teichmüller curve $\mathcal{G}(Y, \alpha)$ is algebraic, see [Moe2, so is the restriction to it of the universal curve. The finite maps $\Phi$ and $\Psi$ preserve algebraicity. Therefore, $\mathcal{G}(X, \omega)$ lies densely on an algebraic surface.

We finish the proof of Theorem 3 with the following. 
Lemma 16. Let $\mathcal{S}$ be the complex algebraic surface of the conclusion of Lemma 15. Each cusp of $\mathcal{G}(X, \omega)$ lies within some two real-dimensional set of $\mathcal{S}$. Let $\Xi$ be the set of infinite ends of $\mathcal{G}(X, \omega)$ corresponding to infinite ends of $P S L(Y, \alpha ; p) \backslash \mathbb{H}^{2}$ covering cusps of $P S L(Y, \alpha) \backslash \mathbb{H}^{2}$. Each infinite end in $\Xi$ lies within some three real-dimensional set of $\mathcal{S}$.

Proof. This follows from Proposition 5 and the finiteness of the various maps: $\pi$, $\Phi, \Psi, f_{p}$ and $\operatorname{PSL}(X, \omega) \backslash \mathbb{H}^{2} \rightarrow \operatorname{PSL}(Y, \alpha ; p) \backslash \mathbb{H}^{2}$.

\section{REFERENCES}

[Ber] J. Bertin, Compactification des schémas de Hurwitz, C. R. Acad. Sci. Paris Ser. I Math. 322 (1996), no. 11, 1063-1066. MR1396641 (97c:14007)

[C] K. Calta, Veech surfaces and complete periodicity in genus 2, J. Amer. Math. Soc., 17 (2004), no. 4, 871-908 (electronic). MR2083470(2005j:37040)

[D] P. Dèbes, Méthodes topologiques et analytiques en théorie inverse de Galois: Théorème d'existence de Riemann, pp. 27-41, in: Arithmétique de revêtements algébriques - Actes du colloque de Saint-Étienne, Sémin. et Congr. 5, Soc. Math. France, Paris, 2001. MR.1924915 (2003h:14046)

[EG] C. J. Earle and F. P. Gardiner, Teichmüller disks and Veech's $\mathcal{F}$ structures, pp. 165-189, in Extremal Riemann surfaces, Contemp. Math. 201, Amer. Math. Soc., Providence, RI, 1997. MR 1429199 (97k:32031)

[EMM] A. Eskin, J. Marklof and D. Morris, Unipotent flows and branched covers of Veech surfaces, e-print. arXiv:math.DS/0408090. To appear, Erg. Th. Dyn. Sys.

[Fo] L. R. Ford, Automorphic Functions, McGraw-Hill, New York, 1929.

[Fr] E. Freitag, Siegelsche Modulfunktionen, Springer, Berlin, 1983. MR0871067 (88b:11027)

[GHS] E. Gutkin, P. Hubert and T. A. Schmidt, Affine diffeomorphisms of translation surfaces: Periodic points, Fuchsian groups, and arithmeticity, Ann. Sci. École Norm. Sup., 4e ser., t. 36 (2003), 847-866. MR2032528 (2004m:37042)

[GJ] E. Gutkin and C. Judge, Affine mappings of translation surfaces: Geometry and arithmetic, Duke Math. J. 103 (2000), 191-213. MR1760625 (2001h:37071)

[Ha] A. Haas, Dirichlet points, Garnett points, and infinite ends of hyperbolic surfaces I, Ann. Acad. Sci. Fenn. Math. 21 (1996), no. 1, 3-29. MR1375503 (97c:30046)

[HM] J. Harris and I. Morrison, Moduli of curves, GTM 187, Springer-Verlag, New York, 1998. MR 1631825 (99g:14031)

[HS1] P. Hubert and T. A. Schmidt, Veech groups and polygonal coverings, J. Geom. Phys. 35 (2000), 75-91. MR.1767943 (2001f:37043)

[HS2] Invariants of translation surfaces, Ann. Inst. Fourier 51 (2001), 461-495. MR 1824961 (2003e:32023)

[HS3] Infinitely generated Veech groups, Duke Math. J. 123 (2004), 49-69. MR 2060022 (2005c:30042)

[Hu] J. Hubbard, Sur les sections analytiques de la courbe universelle de Teichmüller, Mem. Amer. Math. Soc. no. 166, Amer. Math. Soc., Providence, RI, 1976. MR0430321|(55:3326)

[KMS] S. Kerckhoff, H. Masur, and J. Smillie, Ergodicity of billiard flows and quadratic differentials, Ann. of Math. 124 (1986), 293-311. MR0855297 (88f:58122)

[KZ] A. Katok and A. Zemlyakov, Topological transitivity of billiards in polygons, Math. Notes 18 (1975), 760-764. MR0399423 (53:3267)

[L] E. Looijenga, Correspondences between moduli spaces of curves, in: Moduli of curves and abelian varieties, pp. 131-150, Aspects Math., E33, Vieweg, Braunschweig, 1999. MR:1722542(2000k:14021)

[Mc1] C. McMullen, Billiards and Teichmüller curves on Hilbert modular surfaces, J. Amer. Math. Soc. 16 (2003), 857-885. MR1992827 (2004f:32015)

[Mc2] Teichmüller geodesics of infinite complexity, Acta Math. 191 (2003), 191-223. MR.2051398 (2005e:32025)

[Mc3] , Dynamics of $S L(2, \mathbb{R})$ over moduli space in genus two, preprint.

[Mc4] Teichmüller curves in genus two: Discriminant and spin, Math. Ann. 333 (2005), 87-130. 
[MSSV] K. Magaard, T. Shaska, S. Shpectorov, H. Völklein The locus of curves with prescribed automorphism group in Communications in arithmetic fundamental groups (Kyoto, 1999/2001) Sūrikaisekikenkyūsho Kōkyūroku No. 1267 (2002), 112-141. MR1954371

[MH] C. Maclachlan, W. Harvey On mapping-class groups and Teichmüller spaces, Proc. London Math. Soc. (3) 30 (1975), part 4, 496-512. MR0374414 (51:10614)

[M] H. Masur, Interval exchange transformations and measured foliations, Ann. Math. 115 (1982), 169-200. MR0644018 (83e:28012)

[MT] H. Masur and S. Tabachnikov, Rational billiards and flat structures, in: Handbook of dynamical systems, Vol. 1A, pp. 1015-1089, North-Holland, Amsterdam, 2002. MR.1928530 (2003j:37002)

[Moe] M. Möller, Teichmüller curves, Galois actions and $\widehat{G T}$-relations, Math. Nachr. 278 (2005), no. 9, 1061-1077. MR2150378

[Moe2] Variations of Hodge structures of a Teichmüller curve, e-print arXiv:math. AG/0401290.

[Na] S. Nag, The complex analytic theory of Teichmüller spaces John Wiley and Sons, Inc., New York, 1988. MR0927291 (89f:32040)

[N1] P. Nicholls, Garnett points for Fuchsian groups, Bull. London Math. Soc. 12 (1980), 216218. MR0572105 (82b:30056)

[N2] The Ergodic Theory of Discrete Groups, London Math. Soc., Lect. Note Series 143, Cambridge Univ. Press, Cambridge, 1989. MR1041575 (91i:58104)

[P] H. Popp, On a conjecture of $H$. Rauch on theta constants and Riemann surfaces with many automorphisms, J. Reine Angew. Math. 253 (1972), 66-77. MR0472842 (57:12531)

[R] H. Rauch, Theta constants on a Riemann surface with many automorphisms, in: Symposia Mathematica, Vol. III (INDAM, Rome, 1968/69) pp. 305-323 Academic Press, London, 1970. MR0260996 (41:5616)

[S] D. Sullivan, On the ergodic theory at infinity of an arbitrary discrete group of hyperbolic motions, in: Riemann surfaces and related topics: Proceedings of the 1978 Stony Brook Conference (State Univ. New York, Stony Brook, N.Y., 1978), pp. 465-496, Ann. of Math. Stud., 97, Princeton Univ. Press, Princeton, N.J., 1981. MR0624833 (83f:58052)

[Th] W. Thurston, Three-Dimensional Geometry and Topology, Princeton Math. Ser. 25, Princeton Univ. Press, Princeton, 1997. MR1435975 (97m:57016)

[Vch1] W. Veech, The Teichmüller geodesic flow, Ann. Math. 124 (1986), 441-530. MR0866707 (88g:58153)

[Vch2] Teichmüller curves in modular space, Eisenstein series, and an application to triangular billiards, Inv. Math. 97 (1989), 553-583. MR1005006 (91h:58083a)

[Vch3] - Geometric realizations of hyperelliptic curves, pp. 217-226, in Algorithms, fractals, and dynamics (Okayama/Kyoto, 1992), Plenum, New York, 1995. MR1402493 (98f:14022)

[Vo] Ya. Vorobets, Plane structures and billiards in rational polygons: the Veech alternative, Russ. Math. Surv. 51 (1996), 779-817. MR1436653(97j:58092)

[W] S. Wewers, Construction of Hurwitz Spaces. Ph.D. Dissertation, Essen University, Germany, 1998. http://www.math.uni-bonn.de/people/wewers/diss.ps

Institut de Mathématiques de Luminy, 163 aV de Luminy, Case 907, 13288 Marseille CEDEX 09, France

Current address: Laboratoire d'Analyse Topologie et Probabilité, Case Cours A, Avenue Escadrille Normandie-Niemen, 13397 Marseille Cedex 20, France

E-mail address: hubert@cmi.univ-mrs.fr

Department of Mathematics, Oregon State University, Corvallis, Oregon 97331

E-mail address: toms@math.orst.edu 